\title{
DAS BEGRIFFSPAAR SIMPLIZITÄT/KOMPLEXITÄT IN DER RHYTHMISCHEN ANALYSE: THEORETISCHE ANNÄHERUNG UND PRAKTISCHE ANWENDUNG
}

\author{
Franz Krieger
}

\section{Problemstellung}

Das in unterschiedlichsten Bereichen verwendete Begriffspaar Simplizität/ Komplexität zählt seit rund 300 Jahren zu den zentralen Parametern musikalischer Reflexion. So war bereits im 18. Jahrhundert Simplizität im Sinne von Fasslichkeit, Unmittelbarkeit, »Einfalt« ein zentrales Thema der Musikästhetik (Mackensen 2000). Komplexität wiederum begegnet uns explizit etwa in musikpsychologischer Forschung (Werbik 1971; Steck/Machotka 1975; Collier/Wright 1995) wie auch in rezenter musikalischer Rhythmusanalyse (Shmulevich/Povel 2000; Pfleiderer 2006; Thul 2008). Noltze (2010) thematisiert nicht zuletzt, ausgehend vom Bereich Musik, die aktuelle gesellschaftspolitische Brisanz der immer mehr Überhand nehmenden Simplifizierung von Bildungsinhalten. Im musikalischen Alltag, d.h. aus der Sicht musizierender Menschen, wird das Begriffspaar Simplizität/Komplexität üblicherweise in einer der Alltagssprache entsprechenden Sinnbelegung angewandt. Wohl selten nur wird dabei unterschieden, dass sich beide Begriffe, Simplizität wie auch Komplexität, in ihrer musikrelevanten Bedeutung aus einem quantitativen wie auch qualitativen Moment zusammensetzen.

Das quantitative Moment fassbar zu machen, ist das geringere Problem, geht es doch um das Abzählen von etwas, das sich wiederholt (Anzahl identischer Akkorde, identischer Rhythmen usw.). Einer tiefer gehenden theoretisch-methodischen Durchdringung bedarf es hingegen, das qualitative Moment zu handhaben. Hier stellen sich vor allem die folgenden Fragen:

1. Welche musikalischen Faktoren bewirken, dass die eine musikalische Erscheinung simpler bzw. komplexer ist als die andere?

2. Lassen sich diese Faktoren in eine graduelle, widerspruchsfreie Anordnung bringen - vom Simplen hin zum Komplexen? 
3. Besitzt eine solche Anordnung aufgrund der zu erwartenden Vielschichtigkeit lediglich theoretischen Gebrauchswert oder lässt sich daraus ein alltagstaugliches, musikanalytisches Instrumentarium herleiten?

\section{Parameterbezogene Differenzierung}

Eine fundierte Untersuchung musikalischen Materials hinsichtlich Simplizität/Komplexität erfordert es, die Gesamtheit der auditiv in Erscheinung tretenden Parameter mit einzubeziehen. Zu diesen Parametern gehören Harmonik, Rhythmik, Melodik, Artikulation, Akzentuierung, Dynamik, Klangfarbe, Instrumentierung, Ensemblegröße, Raumakustik, variative Interpretation, Improvisation, musikalische Interaktion, musikelektronische Besonderheiten (z.B. Sampling-Einspielungen, Echtzeit-Loops, Verzerrung) u.a.m. Im musikalischen Alltag treten diese Parameter in Kombinationen auf, deren Anzahl entsprechend groß ist. Sie erhöht sich nochmals, wenn dem Rechnung getragen wird, dass es wiederum innerhalb jedem der Parameter eine Reihe von Möglichkeiten gibt (unterschiedliche Akkorde, unterschiedliche Rhythmen usw.). Schließlich ist jene zusätzliche Erweiterung der Möglichkeiten mit einzubeziehen, die sich aus den Interdependenzen diverser Elemente ergibt (z.B. hängt die Dissonanzwirkung auch von der verwendeten Lautstärke $a b)$.

Die letztlich daraus resultierende Anzahl kombinatorischer Möglichkeiten stellt klar, dass ein umfassender Katalog qualitativer musikalischer Kriterien, der diese graduell ordnet - vom Simplen zum Komplexen -, erst gar nicht in Angriff genommen zu werden braucht. Zu umfangreich und zu fein differenzierend wäre das Unterfangen und somit untauglich für den musikologischen Alltag. Ein brauchbarer Weg zeichnet sich jedoch in einem selektiven Vorgehen $\mathrm{ab}$, indem man nicht sämtliche vorkommenden Parameter in die qualitative Sichtung mit einbezieht, sondern nur die für ein Stück wichtigsten. Diese wichtigsten Parameter untersucht man nun hinsichtlich ihrer wesentlichen gestalterischen Faktoren. Als Ergebnis wird der ohnehin vorhandene Gesamteindruck (»eher simpel«, »außergewöhnlich komplex« usw.) spezifiziert, indem man die relevanten Parameter nennt (»in der Harmonik«, »im Offsetting der Bassdrum« usw.) und sie näher beschreibt (»hochalterierte Undezim in der Mittelstimme«, »Simultaneität von Dreierund Vierermetrum« usw.).

Dieses selektive Vorgehen wird hiermit zur Diskussion gestellt. Vom Umfang her scheint es sinnträchtig, inhaltlich bedarf es jedoch noch umfangreicher Überlegungen. Vor allem wird zu klären sein, woraus sich die Wich- 
tigkeit der Parameter wie auch deren wesentliche gestalterische Faktoren definieren. Nachfolgend wird anhand der Rhythmik ein Beispiel dafür skizziert, wie Theorie und Praxis qualitativen Bewertens aussehen könnten. Der theoretische Abschnitt bringt hierbei auf den Punkt, was sich aus heutiger, üblicher musikalischer Praxis ableitet. Mittels der sich anschließenden Exempla wird die Praxistauglichkeit dieser theoretischen Vorüberlegungen getestet.

\section{Rhythmik: Theoretische Annäherung}

1. Rhythmus ist das Produkt aus zeitlichem Verlauf und stattfindendem oder nicht stattfindendem auditivem Ereignis. Das auditive Ereignis kann Geräusche bzw. Tonhöhen beinhalten, wobei letztere hinsichtlich einer rein rhythmischen Betrachtung zumeist irrelevant sind. Es geht somit im Wesentlichen darum, Impakt und Nonimpakt im Sinne tonhöhenloser Idealzustände zu unterscheiden.

2. Die qualitative Differenzierung von Rhythmen fußt auf der Spannung zwischen Impakten und Nonimpakten. Das Ausmaß dieser Spannung resultiert aus der Platzierung der Impakte und Nonimpakte innerhalb des übergeordneten Bezugssystems. Unser wichtigstes übergeordnetes Bezugssystem ist der Takt, der sich durch eine bestimmte Länge und eine bestimmte Betonungshierarchie (betonte/unbetonte Taktteile) definiert.

3. Impakte auf betonten Taktzeiten induzieren geringere Spannung, solche auf unbetonten Taktzeiten größere Spannung. Ebenfalls ist die Spannung umso niedriger, je näher ein Impakt am Beginn eines Taktes liegt, und umso größer, je näher er an dessen Ende platziert ist.

4. Je singulärer ein Impakt auftritt, desto wirkungsvoller erscheint er. Umgekehrt ist die Wirkung einer Pause (= Nonimpakt oder Folge mehrerer Nonimpakte) umso größer, je länger sie ist.

5. Die größte rhythmische Spannung ergibt sich durch Synkopen. Von einer Synkope ist zu sprechen, wenn ein Impakt auf einer unbetonten Taktzeit eintritt und ihm in seiner Wirkung zumindest bis einschließlich der nächsten, darauf folgenden betonten Taktzeit Raum gelassen wird.

6. Ungerade Taktarten vermögen aus ihrer inhärenten Betonungsstruktur heraus mehr rhythmische Spannung zu induzieren als gerade Taktarten. Analog gilt dies für ternäre Beatunterteilung im Vergleich zu binärer.

7. Rhythmische Irritation entsteht, wenn dem Hörer Orientierungspunkte entzogen oder diese verschoben werden. In einem Ausmaß angewandt, 
dass dem Hörer dennoch immer wieder Orientierung möglich ist, erreicht man sehr hohe Spannung bei durchaus gegebener Akzeptanz. In einem zu großen Ausmaß verwendet, finden wir uns in derselben Situation wie bei aleatorischen rhythmischen Ereignissen: Die besonders hohe Spannung geht einher mit einer sehr geringen Akzeptanz.

8. Die qualitative Wahrnehmung rhythmischer Geschehnisse ist tempoabhängig: Je rascher die Geschwindigkeit (d.h. je größer die rhythmische Dichte), desto komplexer der Eindruck. Zunehmende Komplexität geht insofern einher mit zunehmend schwindender mentaler Fassbarkeit.

9. Übliche Rhythmik tritt auf Basis eines (imaginären) regelmäßigen Rasters (Pulsraster) in Erscheinung. Bei kontinuierlichen Tempoänderungen (accelerando, decelerando, tempo rubato) bleiben für den Hörer Pulsraster und rhythmische Gestalt weiterhin erkennbar, jedoch entsteht umso größere Spannung, je rascher eine Tempoänderung geschieht. Sprunghafte Tempoänderungen erschaffen besonders hohe Spannung, sie finden jedoch umso weniger Akzeptanz, je häufiger sie auftreten und je größer die Tempounterschiede sind.

10. Übliche rhythmische Spannung beinhaltet in relativ hohem Maße Wiederholung. Diese kann motivisch-segmentiv erfolgen oder auch in entsprechend längeren Ausformungen (z.B. Rhythmusformeln wie Rumba, Samba usw.). Beim Hörerpublikum befriedigt solcherlei Wiederholung eine gewisse Erwartungshaltung, weswegen aleatorische rhythmische Ereignisse trotz ihrer besonders hohen Spannung keine nennenswerte Akzeptanz finden (vgl. Huron 2006).

\section{Beispiele qualitativer Bewertungen im Bereich der Rhythmik}

Die nachfolgenden Musikbeispiele waren im Juni 2011 Nummer-eins-Hits aus den folgenden Charts: Billboard Hot 100, Billboard Jazz Songs, Billboard Rap Songs und European Top 100 Singles. Ergänzt werden sie um das Siegerstück aus dem Eurovision Song Contest 2011. Von jedem dieser fünf Stücke wurde eine musikalisch prägnante Passage einer Höranalyse unterzogen, um sodann die auffälligsten Charakteristika zu benennen und das rhythmische Geschehen qualitativ zu bewerten. 
(1) Billboard Hot 100: »Rolling In The Deep« (Adele)

(Untersuchter Abschnitt: 0:04-0:13 min.; Instrumentierung: Gesang, Gitarre)

- Auffallend: Rhythmische Irritation in Gesang und Gitarre.

- Qualitative Bewertung: Die rhythmischen Irritationen in Gesang und Gitarre bilden sich bei einer rein rhythmischen Betrachtung (Impakt/Nonimpakt) nicht ab, da sie sich beim Gesang aus dem Text und bei der ostinat in Achteln begleitenden Gitarre aus den Harmoniewechseln ergeben:

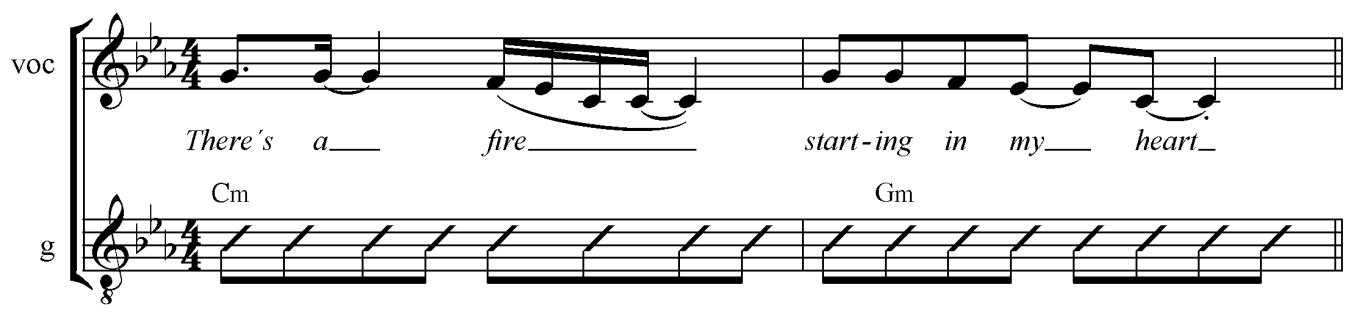

Notenbeispiel 1: Adele - »Rolling In The Deep«

Von komplexer Wirkung ist überdies die immer wieder synkopiert ausgeführte Sechzehntelunterteilung des Viertelbeats in der Gesangsstimme, wodurch sich teilweise ragtimeartige Rhythmusfiguren (z. B. Scotch Snap) ergeben.

(2) Billboard Jazz Songs: »Botswana Bossa Nova« (David Benoit)

(Untersuchter Abschnitt: 0:43-0:48; Instr.: Klavier, Bass, Schlagzeug)

- Auffallend: Durchbrechen der üblichen 4/4-Bewegung mittels einer häufig verwendeten Secondary-Rag-Figur.

- Qualitative Bewertung: Zur relativ komplexen Rhythmik kommt es primär durch Bimetrik, indem Klavier und Bass plötzlich die Secondary-RagFigur spielen, zugleich aber das Schlagzeug weiterhin in gewohnter Weise begleitet. Verstärkt wird diese Gegensätzlichkeit dadurch, dass in harmonischer Hinsicht im Gleichtakt mit dem Rhythmus der SecondaryRag-Figur innerhalb des Quartenzirkels weitergeschritten wird:

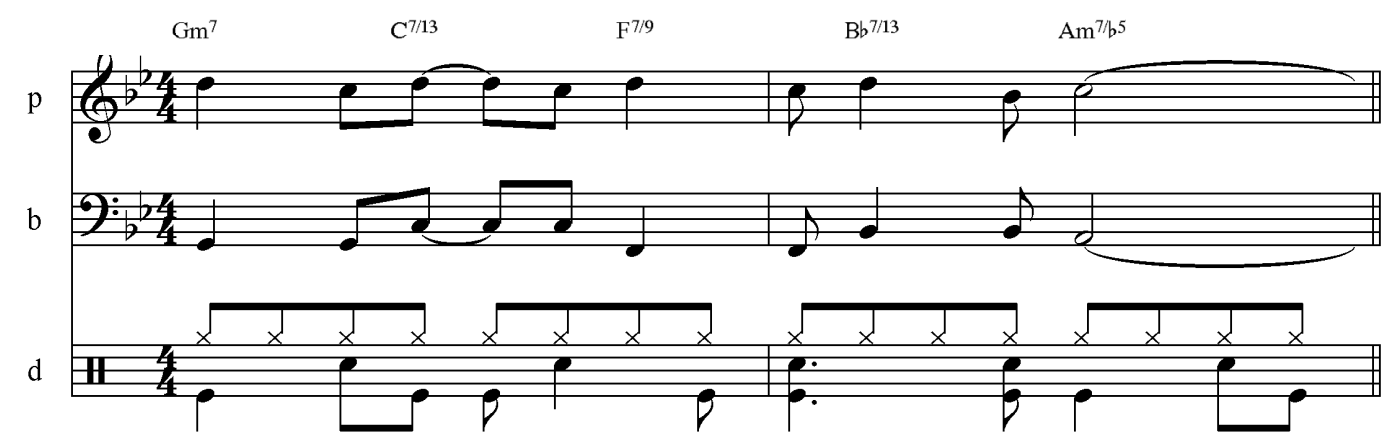

Notenbeispiel 2: David Benoit - »Botswana Bossa Nova« 
(3) Billboard Rap Songs: »Look At Me Now« (Chris Brown feat. Lil Wayne \& Busta Rhymes)

(Untersuchter Abschnitt: 1:16-1:22; Instr.: Rapping, Synthesizer, Schlagzeug)

- Auffallend: Das musikalische Hauptanliegen ist die Sprechkunst, darüber hinaus geht es um den Gegensatz von menschlicher Stimme und der soundmäßigen Künstlichkeit der Begleitung.

- Qualitative Bewertung: Die rhythmische Komplexität ergibt sich aus der Simultaneität dreier metrischer Ebenen: der Vokalstimme (vorwiegend in Sechzehnteln), dem Schlagzeug (binäre Unterteilung des Viertelbeats plus Synkopierung) und dem Synthesizer-Vamp (Synkopierung bezogen auf Halbe Noten):

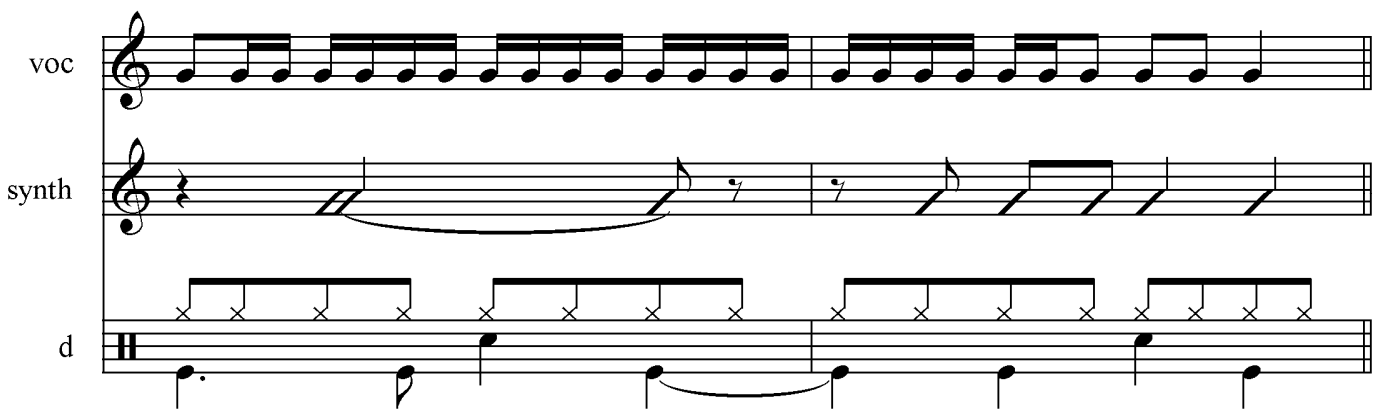

Notenbeispiel 3: Chris Brown ft. Lil Wayne \& Busta Rhymes - »Look At Me Now«

Die Synthesizer-Stimme stellt den wesentlichen Gegensatz zum Rapping dar, da sie sich pantonaler Melodik (in der Art aleatorischer Portamenti) bedient, jedoch immer nur für begrenzte Zeit und Wiederholungen nutzend.

(4) European Top 100 Singles: »Give Me Everything (Pitbull feat. Ne-Yo...) (Untersuchter Abschnitt: 0:45-1:00; Instr.: Gesang, Keyboards, Bass, Schlagzeug)

- Auffallend: Tanzinduzierende, wuchtige, ostinate Rhythmik (Loop-Charakter), relativ wenige unterschiedliche, jedoch ostinate Synkopierungen.

- Qualitative Bewertung: Der Eindruck von Komplexität ergibt sich zuvorderst als Summationsrhythmus von Gesangsstimme (binäre Unterteilung des Viertelbeats plus sparsame Synkopierung) und dem in Sechzehnteln realisierten Keyboard-Vamp (ebenfalls mit wenig Synkopierung):

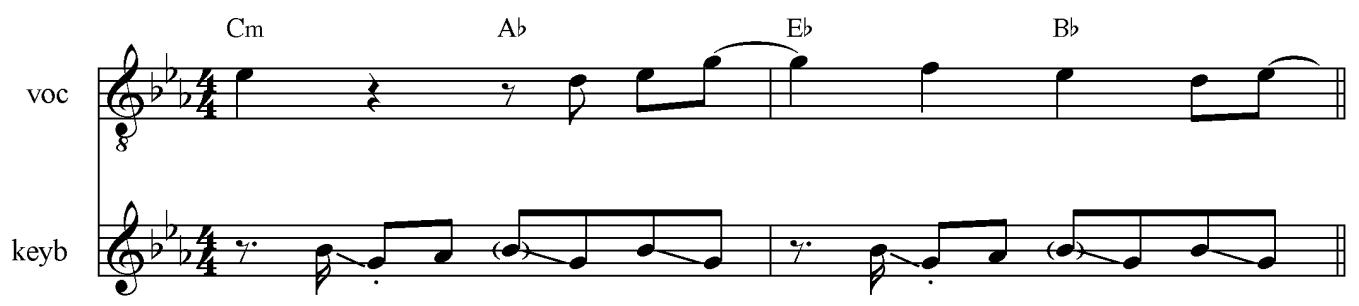

Notenbeispiel 4: Pitbull feat. Ne-Yo, Afrojack \& Nayer - »Give Me Everything « 
Ergänzend findet sich Synkopierung in den respondierenden Background-Vocals, im Bass sowie im Backbeat der Hi-Hat. Das wuchtige Element ergibt sich in rhythmischer Hinsicht aus dem ostinaten Onbeat der Bass Drum, unterstützt von dem sich lautstärkemäßig in den Vorgrund drängenden Keyboard sowie dem Bass.

(5) Eurovision Song Contest 2011: »Running Scared« (Eldar \& Nigar)

(Untersuchter Abschnitt: 0:14-0:25; Instr.: Gesang, Keyboards, Bass, Schlagzeug)

- Auffallend: Extrem dicht instrumentiert (Vielzahl an Instrumentalstimmen; Viertelbeat in Sechzehntelunterteilung); $\mathrm{C}-\mathrm{C} / \mathrm{B}-\mathrm{Am}-\mathrm{F}$ als vertrautes harmonisches Klischée; synkopierter Gesangsvamp.

- Qualitative Bewertung: Der relativ komplexe Eindruck resultiert aus der rhythmischen Dichte, zu der insbesondere der synkopierte Gesangsvamp (Achtelunterteilung des Viertelbeats) sowie die akkordischen Keyboardstimmen (Sechzehntelunterteilung) beitragen. Jedes der rhythmischen Elemente (Gesang, Keyboards, Bass, Schlagzeug) gestaltet zum Großteil mittels Wiederholung. Besondere Spannung ergibt sich aus dem Gesang, indem die (anzunehmende) Erwartungshaltung des Hörers an zwei Stellen nicht erfüllt wird:

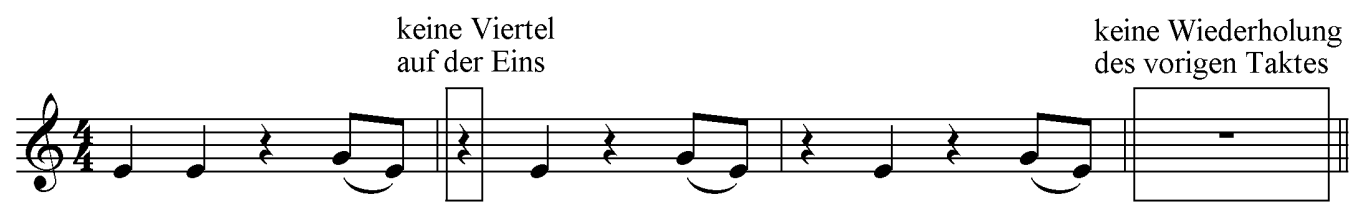

Notenbeispiel 5: Eldar \& Nigar - »Running Scared«

\section{Diskussion und Fazit}

Die Resultate aus den Rhythmusuntersuchungen sind in Präzision und inhaltlicher Tiefe vielversprechend und legen nahe, dass der theoretische Rahmen für die qualitativen Bewertungen in einer passenden Größe gezogen wurde. Zugleich ergeben sich aus der praktischen Auseinandersetzung mit dem musikalischen Material folgende Implikationen bzw. Überlegungen:

(a) Zu den Stärken der vorliegenden theoretischen Betrachtung zählt, dass sie zwecks Alltagstauglichkeit sehr schlank gehalten ist. Ihr Anwenderkreis ist daher jedoch von vornherein auf ein Fachpublikum beschränkt.

(b) Die vorgestellten musikalischen Exempla haben Beispielsfunktion, allerdings können sie nicht unreflektiert als Beleg für die breite Anwendbarkeit des Analysemodells gelten, da der Autor für die Analysen mehr an 
Wissen einfließen lassen musste, als die theoretische Betrachtung bietet. Diese Crux des schlank gehaltenen Instrumentariums ist systemimmanent.

(c) Das quantitative Moment in der Bewertung rhythmischer Geschehnisse wurde bewusst ausgeklammert. In der musikalischen Realität sind Qualität und Quantität jedoch koexistent, daher müssen in eine Analyse letztlich beide Parameter einfließen.

(d) Wiederholung äußert sich nicht nur als Duplizierung, sondern sie ist im musikalischen Alltag auch in variativer Unschärfe vorhanden, d.h. qualitative Erscheinungen wie Gleichförmigkeit oder Abwechslung sind dem Parameter Wiederholung hinzu zu rechnen.

(e) Die grundsätzliche Unterscheidung von Impakt und Nonimpakt ist von melodischen und perkussiven Erscheinungen auf Betonungsphänomene (sprachliche, harmonische, tonhöhenmäßige u.dgl.) hin auszudehnen.

(f) Es gilt anhand weiterer Rhythmusuntersuchungen zu klären, ob die Bereiche Metrik, Rhythmusformeln und Mikrorhythmik explizit in die theoretische Betrachtung einfließen sollen.

Alles in allem steht der Stärke des schlanken Instrumentariums vor allem zweierlei Gefahr gegenüber: Zum einen ist der Katalog der thematisierten Parameter eingeschränkt, es tritt zur willkommenen Unschärfe also das Gespenst der Lücke hinzu. Zum anderen mag bei manchem Anwender der Eindruck entstehen, dass hinter gewissen Schlagworten (z.B. Synkopierung) inhaltlich-qualitativ tatsächlich nicht mehr steht, als in wenigen Sätzen zusammengefasst wird. In diesem Fall hätte man das sprichwörtliche Bad mitsamt dem Kinde ausgeschüttet. Die erläuterten Thesen können somit kein Ersatz sein für ein umfassendes Lehrwerk rhythmischer Analyse.

\section{Literatur}

Billboard-Charts. http://www.billboard.com (Zugriff: 23.6.2011).

Collier, Geoffrey L. / Wright, Charles E. (1995). »Temporal rescaling of simple and complex ratios in rhythmic tapping. « In: Journal of Experimental Psychology: Human Perception and Performance 21, H. 3, S. 602-627.

European Top 100 Singles Charts. http://www.worldcharts.co.uk/world\%20charts/ european.htm (Zugriff: 23.6.2011).

Huron, David (2006). Sweet Anticipation. Music and the Psychology of Expectation. Cambridge, MA, und London: MIT.

Mackensen, Karsten (2000). Simplizität. Genese und Wandel einer musikästhetischen Kategorie des 18. Jahrhunderts. Kassel: Bärenreiter. 
Noltze, Holger (2010). Die Leichtigkeitslüge. Über Musik, Medien und Komplexität. Hamburg: Körber-Stiftung.

Pfleiderer, Martin (2006). Rhythmus. Psychologische, theoretische und stilanalytische Aspekte populärer Musik. Bielefeld: Transcript.

Shmulevich, Ilya / Povel, Dirk-Jan (2000). „Complexity Measures of Musical Rhythms. «In: Rhythm Perception and Production. Hg. v. Peter Desain und Luke Windsor (= Studies on New Music Research [3]). Lisse: Swets \& Zeitlinger, S. 239-244.

Steck, Loren / Machotka, Pavel (1975). »Preference for musical complexity: Effects of context. «In: Journal of Experimental Psychology: Human Perception and Performance 1, H.2, S. 170-174.

Thul, Eric (2008). »Measuring the Complexity of Musical Rhythm. « Master thesis. Montreal: McGill University, http://cgm.cs.mcgill.ca/ godfried/teaching/mirreading-assignments/Eric-Thul-Thesis.pdf (Zugriff: 27.9.2011).

Werbik, Hans (1971). Informationsgehalt und emotionale Wirkung von Musik. Mainz: Schott.

\title{
Diskographie
}

Adele (2011). »Rolling In The Deep. « Auf: 21. XL Recordings 44699.

Benoit, David (2010). »Botswana Bossa Nova." Auf: Earthglow. HeadsUp HUI 3197502.

Brown, Chris, feat. Lil Wayne \& Busta Rhymes (2011). »Look At Me Now. Auf: F.A.M.E. Jive 86067.

Eldar \& Nigar (2011). »Running Scared.« Auf: Eurovision Song Contest Düsseldorf 2011. CMC 0970562.

Pitbull feat. Ne-Yo, Afrojack \& Nayer (2011). »Give Me Everything.« Single. RCA G0100024690404.

\begin{abstract}
In musical analysis the conceptual polarity of simplicity and complexity is usually used in its everyday definition. This paper extends this definition by differentiating a quantitative meaning of complexity from a qualitative one. The substantial investigation of the latter is usually aggravated by the simultaneity of the multitude of musical parameters, creating a myriad of interdependences. Thus it is proposed to segment music into its most important parameters and to create a separate analytical framework for each of them. This theoretical approach is exemplified by the parameter of rhythm.
\end{abstract}

